\title{
Prognostic Factors in Patients with Diffuse Type Gastric Cancer (Linitis Plastica) after Operative Treatment
}

\author{
M. Schauer ${ }^{1}$, M. Peiper ${ }^{1}$, J. Theisen ${ }^{2}$, W. Knoefel ${ }^{1}$ \\ ${ }^{1}$ Department of General-, Visceral-, and Pediatric Surgery, Universitaetsklinikum Duesseldorf, Heinrich Heine Universitaet, \\ Duesseldorf, Germany \\ ${ }^{2}$ Department of General- Visceral and Thoracic Surgery, Technische Universitaet Muenchen, Germany
}

\begin{abstract}
Objective: Treatment options for patients with diffuse type gastric cancer (linitis plastica) are discussed controversial. It is sometimes discussed that these patients should be treated primarily in palliative intention conservative without resection.

Methods: In a single-center analysis, we investigated 120 patients with diffuse type gastric cancer. All patients underwent a total gastrectomy, 45 patients even a multivisceral resection because of infiltrating growth, or metastases. Serum tumor marker CEA, CA 72-4, and CA 19-9 were recorded in all patients before surgery. An immunocytochemical detection of free peritoneal tumor cells (FPTC) using Ber-EP4 antibody was correlated with tumor stage and survival. Median follow-up time was 38 months.

Results: Complete resection rate was 31\% ( $\mathrm{n}=37$ ). $61 \%(\mathrm{n}=73)$ of all patients had already distant metastases at the time of surgery, $80 \%$ of them peritoneal carcinomatosis. Median survival for the whole group was 8 months, after complete resection 17 months. Lavage cytology, distant metastases, resection rate, and CA19-9 levels had significant influence on survival. Conclusion: A significant survival advantage for patients with diffuse type gastric cancer can only be achived after complete resection. We could define a subset of patients with an extremely poor prognosis even after surgical resection. Meticulous preoperative staging, including a diagnostic laparoscopy to exclude peritoneal carcinomatosis and free peritoneal tumor cells before resection should be mandatory in these patients.
\end{abstract}

Key words: gastric cancer; prognostic factors; diffuse typ gastric cancer; linitis plastica; free peritoneal tumor cells

\section{INTRODUCTION}

Patients with linitis plastica of the stomach, have a poor prognosis with a five year survival of $3-10 \%$ in various studies [1, 2]. Having this detrimental prognosis in mind, controversy in treatment of these patients still exists. The question is whether surgical resection is suitable for these patients or to begin with a nonoperative treatment [3-6].
Since peritoneal seeding, extended lymphnode metastasis and the extension of the tumor into the neighbouring organs are relatively common, only about $20 \%$ of the patients with linitis plastica benefit from total gastrectomy [7,8]. Since a complete resection is not possible in the majority of patients with linitis plastica of the stomach, the result of a postoperative radiation with or without chemotherapy or neoadjuvant approaches have been discussed in the last couple of years [8-10]. Patients with a diffuse type gastric cancer with a signet ring appearance are known to show very little response rates on a multimodal treatment with chemo- or radio-chemotherapy [5-11].

The linitis plastica represents $7-10 \%$ of gastric adenocarcinomas in its typical ,signet ring“-form [2]. As few reports have focused specifically on the treatment, staging and prognosis of this entity, the purpose of this study was to specify its clinical features, treatment options and prognostic factors for a more individually tailored treatment.

\section{PATIENTS AND METHODS}

A review of our prospective database for patients operated on for gastric carcinoma identified 120 patients with linitis plastica, representing $8,6 \%$ of all patients with gastric cancer operated on during a ten year time period $(n=1396)$.

By definition of linitis plastica on the postoperative histopathological specimen all included patients presented with a locally advanced tumor stage (pT3 or pT4 category), and diffuse infiltration of neoplastic signet ring cells. All tumors belonged to the diffuse type according to the Lauren classification. The macroscopic appearance was classified during preoperative endoscopy as Borrmann type III or IV. We excluded patients with a palliative chemotherapy without a following resection. Follow-up was complete for all patients. The median follow-up time was 38 months (range 2 to 120 months).

Standardized staging procedure was performed in all patients including endoscopy, endosonography, abdominal ultrasound, and a computed tomography scan. The serum tumor markers CEA, CA 19-9 and CA 72-4 were obtained in all patients before treatment. A diagnostic laparoscopy was performed in 70 
patients. During this procedure an abdominal lavage was obtained. Immediately after opening the abdominal cavity $500 \mathrm{ml}$ saline was introduced into the upper abdomen followed by a collection of five samples of $60 \mathrm{ml}$ each from the subphrenic space. Centrifugation steps, slide preparation and immunocytochemical staining were performed as described previously [12]. The monoclonal antiepithelial non-cytokeratin antibody Ber-Ep4 (Dako, Hamburg, Germany) used for immunocytochemical staining shows a broad pattern of reactivity with the majority of epithelial tissues and labels carcinoma cells. Conventional cytological criteria of malignancy were required to be present in a BerEp4-positive cell [13]. All slides were evaluated by two independent reviewers.

All patients underwent a total $(\mathrm{n}=75)$ or an extended $(n=45)$ gastrectomy including a lymphadenectomy of compartments I and II by en bloc resection (D2 lymphadenectomy). A multivisceral resection with partial resection of the pancreas, liver, colon or spleen had to be performed in 10 patients because of infiltrative growth. In 43 patients an extended resection had to be performed for distant metastases. In 40 of these cases a circumscribed peritoneal carcinomatosis and in 12 patients small liver metastases could be resected. Pathohistological work-up with depth of invasion, nodal involvement, distant metastasis, and resection status were recorded according to the UICC 2002 [14].

Follow-up was performed in our outpatient oncology clinic according to a standard protocol. All patients presented every three months during the first year and every six months the following years. Patients underwent a clinical examination, ultrasound, a CT scan and an endoscopy every time.

Statistics: Data are expressed as median values and ranges. Survival rates were calculated according to $\mathrm{Ka}$ plan-Meier for various groups and compared by logrank test. Survival data is given as observed overall survival and include postoperative deaths. The tumor markers CEA, CA 19-9 and CA 72-4 were coded as binary variables by employing log-rank statistics to determine optimal cut-off points for discrimination of low- and high-risk patients. The prognostic impact of all analyzed factors on survival time after resection was assessed by the Cox's Proportional Harzard Model using the SPSS software package (SPSS INC. Chicago, IL, USA) which provides estimates of the relative risk together with $95 \%$ confidence intervals. Significance was assessed by chi-square analysis. For comparison of prognostic strength of the various factors, covariate selection was carried out by multivariate regression by a stepwise forward procedure which chooses factors with the highest additional prognostic information. All test decisions were performed at a significance level of $\mathrm{p}<0.05$.

\section{RESULTS}

One hundred and twenty patients with linitis plastica were included in this study. Mean age was 57.7 years (range from 28 to 83 years). The male to female ratio was 1:1.

The preoperative staging showed the main localisation of the diffuse gastric carcinoma in the antrum and the pylorus $(41.4 \%)$, in the corpus $(33.6 \%)$ or the fundus $(24.1 \%)$. Endoscopically $14.2 \%$ of all patients showed a Borrmann type III, 75.8\% a Borrmann typ IV tumor.

Elevated serum levels of CEA, CA 19-9 and CA $72-4$ were found in $25.2 \%, 30 \%$, and $39 \%$, respectively. CEA and CA 72-4 did not have a significant influence upon survival, tumor localisation, morphology, tumor mass, serosal infiltration or metastatic status. A log-rank statistic showed that high values of CA 19-9 (cut off level $>45 \mathrm{U} / \mathrm{l}$ ) is correlated with a worse 5year survivalrate $(p=0.007)$ (Fig. 1.). A correlation between the pTNM-category and high CA 19-9 values could not be confirmed.

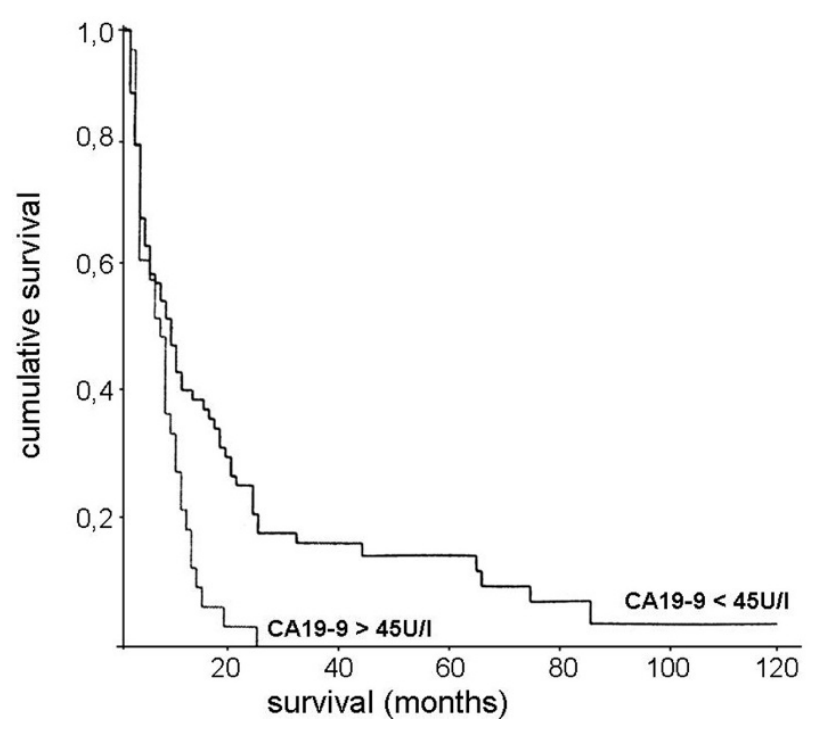

Fig. 1. Cumulative survival of patients with diffuse type gastric carcinoma after surgical resection. Comparison of patients with high and low preoperative serum levels of CA19-9 (cut-off $45 \mathrm{U} / \mathrm{l}$ ). $\mathrm{p}=0.007$

The diagnostic laparoscopy could add valuable information about the tumor stage as a very safe procedure. There did neither occur any postoperative complication nor an additional tumor spread in all 70 patients. Especially the local depth of invasion (T3- vs. T4-category) could very well be determined. With laparoscopy or laparotomy at the beginning of gastrectomy, peritoneal carcinomatosis could often easily be visualized and confirmed by biopsy in 33\% of all patients. Hepatic metastasis could be demonstrated with intraoperative ultrasound and biopsy in 10\% of patients.

The investigation of the lavage fluid revealed in $26.7 \%$ malignant tumor cells (FPTC). In patients with a negative cytology, 5-year-survival rate was $20 \%$ compared to $2 \%$ in patients with a positive cytology (median survival 13 vs. 3 months, $\mathrm{p}<0,001$ ) (Fig. 2.). A significant correlation between FPTC and peritoneal carcinomatosis or the pTNM-category could not be found. In 11 cases $(15.7 \%)$ a positive cytology could be found without any sign of peritoneal or distant metastasis. In 12 cases with initially negative lavage cytology we found a peritoneal carcinomatosis at the time of gastrectomy. 


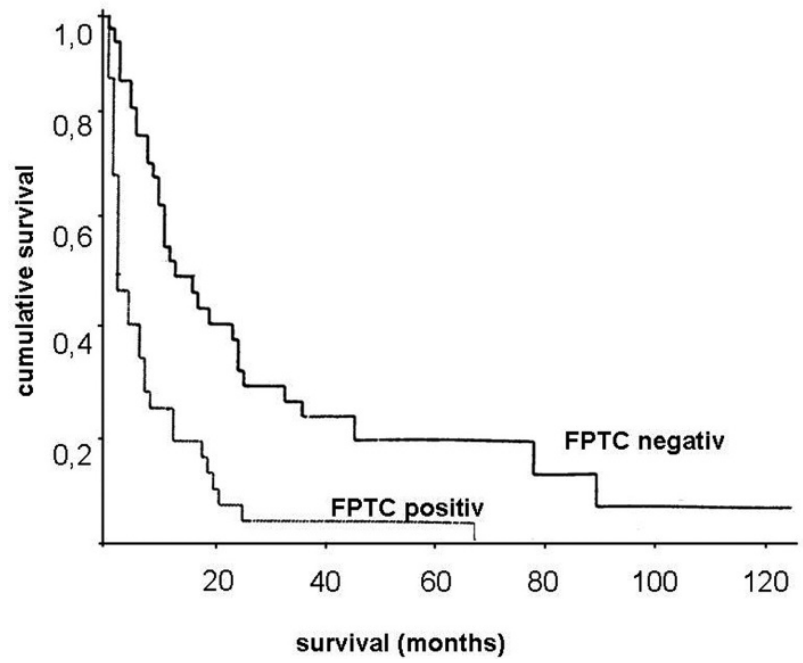

Fig. 2. Cumulative survival of patients with diffuse type gastric carcinoma after surgical resection. Comparison of patients with versus without immunocytological detection of free peritoneal tumor cells before surgery. $\mathrm{p}<0.001$

After the preoperative staging procedure all patients underwent a resection; a total gastrectomy was performed in $61.7 \%$ of the patients, a transhiatal gastrectomy was done in $22.5 \%$ of the cases. In patients with a pT4 gastric cancer with invasion of neighbouring organs $(8.3 \%)$ a multivisceral resection (stomach, transverse colon, spleen, or pancreas) was performed. A palliative subtotal gastrectomy due to a malignant gastric outlet syndrome was done in $7.5 \%$ of the patients. The type of resection did not have a significant influence on the postoperative morbidity or mortality. All patients after a multivisceral resection survived at least 30 days. After 90 days three of these patients $(37.5 \%)$ had died compared to 44 deaths in the whole investigated group $(36 \%)$.

The pathohistological work-up (according to the UICC2002 [14]) is summarized in Table 1. 60.8\% of all patients had distant metastasis. In $80 \%$ of these patients a peritoneal carcinomatosis could be diagnosed. Complete resection was possible in 31\% $(n=37), 69 \%$ of the patients had residual disease.

The median overall survival time of all patients with linitis plastica investigated was 8 months; five year survival 8\% (Fig. 3). Patients, who underwent a complete tumor resection (R0), had a median survival time of 17 months (range 13 to 21 months), while patients with a microscopic (R1) or a macroscopic (R2) residual tumor showed a median survival time of only 6 months (range 4 to 8 months, $(p<0,001)$ (Figure 3.). There was no difference in survival after a R1- and a R2-resection. In patients with a peritoneal carcinomatosis or some other kind of distant metastasis, survival time was significantly worse $(\mathrm{p}<0,001)$; median survival was 16 (M0) (range 8 to 24 ) versus 6 (M1) (range 4 to 8 ) months.

Following univariate analysis complete resection, a negative lavage cytology, high CA 19-9 levels and the presence of distant metastasis had significant influence on survival. In this study we did not find an influence of sex, age, localisation of the tumor and

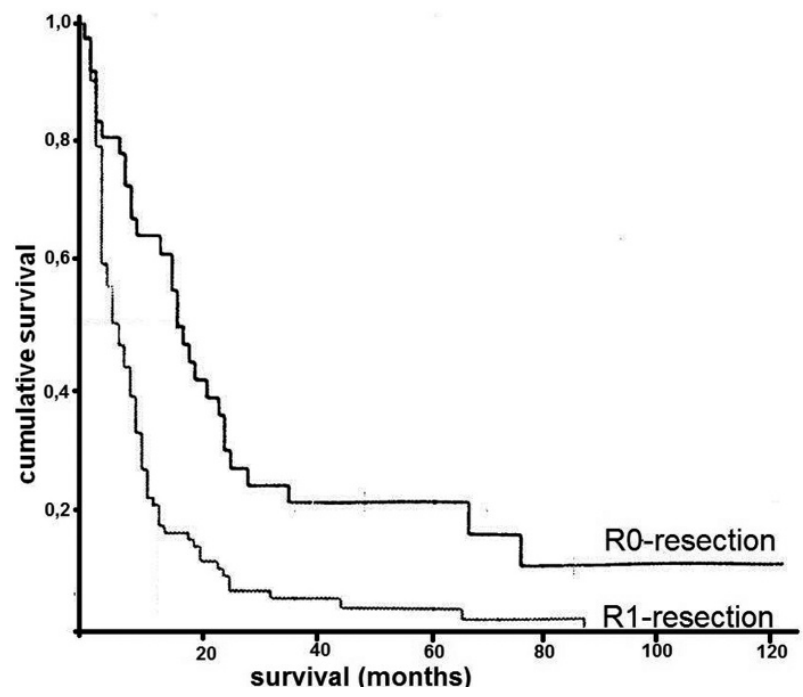

Fig. 3. Cumulative survival of patients with diffuse type gastric carcinoma after surgical resection. Comparison of patients after complete resection and patients with residual disease. $\mathrm{p}<0.001$

Table 1. Classification of diffuse type gastric carcinoma in 120 patients according to the UICC 2002.

\begin{tabular}{llll}
\hline & & patients $(\mathrm{n}=120)$ & patients $(\%)$ \\
\hline pT category & T3 & 82 & 65 \\
& T4 & 38 & 35 \\
pN category & N0 & 15 & 12.5 \\
& N1 & 23 & 19.2 \\
& N2 & 22 & 18.3 \\
& N3 & 60 & 50 \\
pM category & M0 & 47 & 39.2 \\
R category & M1 & 73 & 60.8 \\
& R0 & 37 & 30.8 \\
& R1 & 38 & 31.7 \\
Grading & R2 & 45 & 37.5 \\
& G2 & 6 & 5 \\
& G3 & 94 & 78.3 \\
& G4 & 20 & 16.7 \\
\hline
\end{tabular}

serum tumor markers like CEA or CA 72-4 upon survival. Following the multivariate analysis, the most significant survival advantage for patients with a linitis plastica of the stomach can be achieved only after complete resection in patients without distant metastasis. Patients with a peritoneal carcinomatosis, a high CA 19-9 level and a positive lavage cytology define a subset of patients with an extremely poor prognosis.

\section{DisCUSSION:}

The term „linitis plastica“ (Greek for linen cloth or net) was introduced by Brinton 1858 on the basis of macroscopic criteria [15]. Up until 1943 it was unclear whether linitis plastica, also called „leather bottle stomach", was an inflammatory or a neoplastic disease, when Saphir described the macroscopic morphology, the neoplastic nature, and the progression 
with metastatic spread of this type of carcinoma [16, 17].

The diffuse type gastric cancer is characterized by infiltration of neoplastic cells with significant desmoplastic response, imparting a rigid, fibrotic appearance to the wall [2]. Histopathologically, linitis plastica consist of diffusely infiltrating, small, atypical cells, that often have a signet ring appearance [18]. They spread early via lymphatic dissemination and by local extension into neighbouring organs or as peritoneal carcinomatosis. Most of our patients showed at the time of diagnosis a lymph node involvement and distant metastasis. $87.5 \%$ of our patients showed lymph node involvement, half had peritoneal implants, in $20 \%$ we saw positive paraaortal lymph nodes counting as distant metastases (M1(lym) according to the UICC [14]). Hematogenous liver metastasis is relatively rare associated with the linitis plastica $(10 \%)$ [18]. In case of liver metastasis, distant lymph node metastases or peritoneal carcinomatosis, our patients showed a median survival of only 6 months. Gastrectomy could only be performed in palliative intention in these patients.

The diffuse type gastric cancer has an extremely bad overall prognosis. The median survival of our patients was only 8 months, five year survival $8 \%$. In order to decide about the adequate treatment modality a meticulous staging procedure is necessary. Our objective was to define prognostic factors in order to exclude patients, who will not have any benefit from primary resection.

The diagnostic laparoscopy turned out to be a very safe procedure for the diagnosis of the local tumor spread, lymph node status, distant metastases, and the histological proof of peritoneal implants. In $60 \%$ of our investigated patients peritoneal seeding could only be diagnosed at the time of laparoscopy, but did not have a CT-correlation [19, 20].

The tumor marker CA 19-9 (cut-off value of $45 \mathrm{U} / \mathrm{l})$ could be identified as a prognostic factor. In our patient collective high preoperative serum levels of CA19-9 were significantly associated with a dismal survival rate. CA 19-9 is known to be elevated in gastric carcinomas with lymph node involvement and peritoneal spread [2]. CA 72-4 is correlated to the tumor mass [21].

The immunocytochemical detection of FPTCs turned out to be an independent additional strong prognostic factor as also described in other tumor entities before [22-24]. The short survival time of these patients is similar to patients with an M1-disease [25, $26]$. There was no correlation to tumor stage or presence of metastases. Surprisingly, FPTCs were not correlated with the existence of peritoneal carcinomatosis.

Peritoneal carcinomatosis is generally assumed to occur as a result of shedding from tumor cells from the serosal surface of the primary tumor or via the lymphatic drainage system $[27,28]$. Lymphoreticular organs, called milkey spots, occur throughout the greater omentum and may represent the barrier to peritoneal seeding [29]. The present data suggest that the release of tumor cells from lymphatic capillaries plays an important role in peritoneal dissemination. The missing FPTCs in patients with already dissemi- nated peritoneal carcinomatosis would suggest that a shedding of tumor cells can be only milde. However, FPTCs without an existing peritoneal carcinomatosis must be regarded as a precursor. Our results show that patients with FPTCs have the same prognosis as patients with distant metastases.

A significant survival advantage for patients with linitis plastica can only be achieved after complete resection $[2,3,18] .31 \%$ of all investigated patients could be completely resected. This subgroup of patients had a median survival of 17 months and did significantly better than patients with residual tumor. A difference in survival between patients with microscopic (R1-) and macroscopic (R2-) residual tumor did not reach statistical significance $[2,18]$. Even patients with a multivisceral resection had a significant survival benefit. Postoperative mortality after gastrectomy and multivisceral resection were equal with the same 30and 90-day survival rate. Similar survival advantages in locally advanced gastric carcinomas treated with a multivisceral resection could also be demonstrated by $\mathrm{Fu}-$ rukawa $[30,31]$.

In conclusion we could define a subset of patients with diffuse gastric cancer, who benefit from a primary surgical resection. The prognosis of patients with diffuse type gastric cancer can only be ameliorated by a complete resection. Therefore, surgery is indicated, if an R0-resection can be anticipated even if a multivisceral resection would be necessary. In terms of a meticulous staging a diagnostic laparoscopy with an abdominal lavage with immunohistological examination for FPTCs to reveal even hidden peritoneal dissemination seems to be mandatory. In presence of peritoneal carcinomatosis or a positive lavage cytology, resection offers no survival advantage.

\section{REFERENCES}

1. Salvon-Harman JC, Cady B, Nikulasson S, Khettry U, Stone MD, Lavin P. Shifting Proportons Of Gastric Adenocarcinomas. Arch Surg. 1994, 129(4): 381-388.

2. Park JC, Lee YC, Kim JH, Kim YJ, Lee SK, Hyung WJ, Noh SH, Kim CB. Clinicopathological aspects and prognostic value with respect to age: an analysis of 3,362 consecutive gastric cancer patients. J Surg Oncol. 2009 Jun 1;99(7):395-401.

3. Kodera Y, Ito S, Mochizuki Y, Yamamura Y, Misawa K, Ohashi N, Nakayama G, Koike M, Fujiwara M, Nakao A. The number of metastatic lymph nodes is a significant risk factor for bone metastasis and poor outcome after surgery for linitis plastica-type gastric carcinoma. World J Surg. 2008 Sep;32(9):2015-20.

4. Rougier P, Mahjoubi M, Lasser P, Ducreux M, Oliveira J, Ychou M, Pignon JP, Elias D, Bellefqih S, Bognel C. Neoadjuvant Chemotherapy in Locally Advanced Gastric Carcinoma - a Phase II Trial with Combined Continuous Intravenous 5-Fluorouracil and Bolus Cisplatinum. Eur J Cancer. 1994, 30A(9): 1269-75.

5. Sasaki T, Koizumi W, Tanabe S, Higuchi K, Nakayama N, Saigenji K. TS-1 as first-line therapy for gastric linitis plastica: historical control study. Anticancer Drugs. 2006 Jun;17(5):581-6.

6. Takahashi I, Matsusaka T, Onohara T, Nishizaki T, Tashiro H, Wakasugi K, Kume K, Maehara Y, Sugimachi K Clinicopathological Features of Long-Term Survivors of Scirrhous Gastric Cancer. Hepatogastroenterology. 2000, 47(35):1485-8. 
7. Siewert JR, Sendler A. Linitis Plastica - to Treat or not to Treat. Mosby Surgery; 1999. Kap. 3.24b

8. Webb A, Cunningham D. Curing gastric cancer--hone the scalpel with magic? Br J Cancer. 1996 Feb; 73(4):418-9.

9. Henning GT, Schild SE, Scott L, Stafford SL. Results of Irradiation or Chemoirradiation Following Resection of Gastric Adenocarcinoma. Int.J. Radiation Oncology Biol. Phys. 2000, 46(3): 589-598.

10. Visset J, Hamy A, Letessier E, Aillet G, Paineau J. Linitis plastica of the stomach. Factors influencing prognosis. Chirurgie. 1992, 118(4): 236-242.

11. Caudry M, Ratoanina JL, Escarmant P, Maire JP. Target Volume in Radiotherapy of Gastric Adenocarcinoma. Cancer Radiother; 2001, 5(5): 523-33.

12. Rosenberg R, Nekarda H, Bauer P, Schenck U, Hoefler $\mathrm{H}$, Siewert JR. Free peritoneal tumour cells are an independent prognostic factor in curatively resected stage IB gastric carcinoma.. Br J Surg. 2006 Mar; 93(3):325-31.

13. Beer TW, Shepherd P, Theaker JM. Ber EP4 and epithelial membrane antigen aid distinction of basal cell, squamous cell and basosquamous carcinomas of the skin. Histopathology. 2000 Sep; 37(3):218-23.

14. Greene FL, Page DL, Fleming ID, Fritz A, Baich CM (eds). AJCC Cancer Staging Manual (6th edn). Springer: New York, 2002.

15. Brinton W. The Disease of the Stomach. 1859. London. 310

16. Moreaux J, Barrat F, Msika S. La linite plastique de l'estomac. Chirurgie. 1986. 112, p.485-492

17. Saphir O, Parker M. Linitis Plastica Type of Carcinoma. Surg, Gyn and Obst. 1943, 76: 206-213

18. Mastoraki A, Papanikolaou IS, Sakorafas G, Safioleas M. Facing the challenge of managing linitis plastica--review of the literature. Hepatogastroenterology. 2009 NovDec;56(96):1773-8.

19. Sotiropoulos GC, Kaiser GM, Lang H, Treckmann J, Brokalaki EI, Pottgen C, Gerken G, Paul A, Broelsch CE. Staging laparoscopy in gastric cancer. Eur J Med Res. 2005 Feb 28;10(2):88-91.

20. Onate-Ocana LF, Gallardo-Rincon D, Aiello-Crocifoglio V, Mondragon-Sanchez R, De-La-Garza-Salazar J. The Role of Pretherapeutic Laparoscopy in the Selection of Treatment for Patients with Gastric Carcinoma: a Proposal for a Laparoscopic Staging System. ANN Surg Oncol. 2001, 8(8):622-3

21. Gaspar MJ, Arribas I, Coca MC, Diez-Alonso M. Prognostic Value of Carcinoembryonic Antigen, CA 19-9, and CA 72-4 in Gastric Carcinoma. Tumour Biol; 2001, 22(5):318-22.

22. Bölke E, Orth K, Gerber PA, Lammering G, Mota R, Peiper M, Matuschek C, Budach W, Rusnak E, Shaikh S, Dogan B, Prisack HB, Bojar H. Gene expression of circulating tumour cells and its correlation with tumour stage in breast cancer patients. Eur J Med Res. 2009 Aug 12;14(8):359-63.
23. Bölke E, Orth K, Gerber PA, Lammering G, Mota R, Peiper M, Matuschek C, Budach W, Rusnak E, Shaikh S, Dogan B, Prisack HB, Bojar H. Gene expression of circulating tumour cells in breast cancer patients. Eur J Med Res. 2009 Sep 28;14(10):426-32.

24. Fujimoto T, Zhang B, Minami S, Wang X, Takahashi Y, Mai M. Evaluation of Intraoperative Cytology for Advanced Gastric Carcinoma. Oncology; 2002, 62(3):201-8.

25. Badgwell B, Cormier JN, Krishnan S, Yao J, Staerkel GA, Lupo PJ, Pisters PW, Feig B, Mansfield P. Does neoadjuvant treatment for gastric cancer patients with positive peritoneal cytology at staging laparoscopy improve survival? Ann Surg Oncol. 2008 Oct;15(10):2684-91. Epub 2008 Jul 23.

26. Bonenkamp JJ, Sasako M, Hermans J, Van De Velde CJ. Tumor Load and Surgical Palliation in Gastric Cancer. Hepatogastroenterology; 2001, 48(41): 1219-21.

27. Maehara Y, Hasuda S, Koga T, Tokunaga E, Kakeji Y, Sugimachi K. Postoperative outcome and sites of recurrence in patients foliowing curative resection ofgastric cancer. BrJ Surg 2000; 87: 353-357.

28. SampsonJA. Implantation peritoneai carcinomatosis of ovarian origin. AmJPatbol 1931; 7: 423—443.

29. Liebermann-Meffert D, White H. The Greater Omentum. Springer: New York, 1983.

30. Dahr DK, Kubota H, Tachibana M, Kinugasa S, Masunaga R, Shibakita M, Kohno H, Nagasue N. Prognosis of T4 Gastric Carcinoma Patients: an Appraisal of Aggressive Surgical Treatment. J Surg Oncol; 2001, 76(4):27882.

31. Furukawa $\mathrm{H}$, Hiratsuka M, Iwanaga T, Ishikawa O, Kabuto T, Sasaki Y, Kameyama M, Ohigashi H, Nakamori S, Yasuda T. Extended Surgery - Left Upper Abdominal Exenteration Plus Appleby's Method - For Type 4 Gastric Carcinoma. Ann Surg Oncol; 1997, 4(3): 209-14.

Received: September 27, 2010 / Accepted: Ocober 20, 2010

Address for correspondence:

M. Schauer, MD

Department of General-, Visceral-, and Pediatric Surgery

Universitaetsklinikum Duesseldorf

Heinrich Heine Universitaet

Moorenstrasse 5

40225 Duesseldorf

Germany

Tel.: $\quad+49-221-81-17350$

Fax: +49-211-81-17359

E-mail: matthias.schauer@med.uni-duesseldorf.de 